\title{
Efecto del nivel proteico dietario sobre el tamaño de camada y ganancia de peso total en crías de cuyes raza andina
}

\author{
Dietary protein effect over litter size and total weight gaining in Andina guinea pig's offspring \\ Carlos Paco $^{1}$, Luisa Echevarría ${ }^{1}$ \\ RESUMEN
}

El objetivo del estudio fue evaluar el efecto de tres niveles dietarios de proteína (12 g/100 g PC, 17g/100g PC y 22 $\mathrm{g} / 100 \mathrm{~g}$ PC) sobre el número de crías nacidas, el peso de la camada al nacimiento y al destete, y la ganancia de peso total de la camada. Se utilizaron cuyes de raza Andina debido a su precocidad y prolificidad. Las hembras recibieron 50 gramos diarios de las dietas experimentales desde 16 días previos al empadre. Durante el empadre, recibieron la dieta experimental solo durante el día $(08: 00$ - 14:00 hrs) por 32 días. Finalmente, fueron alimentadas con la misma ración experimental desde la gestación hasta la época del destete (14 días postparto). Los resultados demostraron que no existe un efecto significativo del nivel proteico de la dieta sobre el número de crías nacidas y pesos totales de camada, sin embargo, para los pesos al destete y ganancia total diaria, la dieta normoproteica (17 g/100 g) ofreció mejores resultados $(\mathrm{p}<0,05)$.

PALABRAS CLAVE: Cobayo, Cavia porcellus, dieta de proteínas, ganancia de peso.

\section{SUMMARY}

The objective was to evaluate of the effect of three different protein levels (12 g/100 gr, $17 \mathrm{~g} / 100 \mathrm{gr}$ and $22 \mathrm{~g} / 100 \mathrm{gr})$ over reproductive parameters: litter size, total litter weight, total litter weaning weight and total weight gains. Andina breed was chosen due to their reproductive characteristics such as precocity and prolificity. Females consumed 50 gr of the experimental diets; initially, for a period of 16 days prior mating, then they were fed only during daylight from 0800 to 1400 hours and mated until the next day. Mating phase lasted 32 days. Then, females received their diets during pregnancy and 14 days after parturition. Results showed no significant difference between mother's dietary protein for litter size and total litter weight, however for the total litter weaning weight and daily weight gain, normoproteic $(17 \mathrm{~g} / 100) \mathrm{g}$ diets showed better results $(\mathrm{p}<0.05)$.

KEYWORDS: Guinea pig, cavia porcellus, protein diet, weight gaining.

\section{INTRODUCCIÓN}

El cuy doméstico (Cavia porcellus) es una especie de alta rentabilidad (196\%), gran adaptación nutricional y tolerancia a ecosistemas agrestes (Chauca, 1997). Se caracteriza por ser prolífico y precoz, alcanzando la pubertad entre los 2 a 3 meses, las hembras son poliéstricas no estacionales, con ciclos estrales de 16 días, ovulación espontánea y gestación de 59 a 72 días (Zaldívar, 1986).

Los parámetros productivos en cuyes podrían alterarse por diversos factores, como la nutrición. Estudios han demostrado que niveles proteicos

Facultad Medicina Veterinaria y Zootecnia, Universidad Peruana Cayetano Heredia. Lima, Perú 
óptimos tienen un efecto positivo sobre pesos al destete, el número de gazapos destetados y las tasas de sobrevivencia (Wheat, Spies, Tran y Kock, 1962; Saravia, Muscari, y Chauca, 1983). Por otro lado, Camolezi (2011) evidenció que la subnutrición y restricciones proteicas en ratonas gestantes desencadenan alteraciones como pesos bajos al nacimiento y bajas tasas de fertilidad. Intuitivamente, estudios demostraron un efecto sobre la programación fetal produciendo consecuencias negativas a largo plazo como: ciclos estrales más largos, mayor tiempo de empadre, menor número de cuerpos lúteos, y menor número de implantaciones (Terry, 2005).

El uso de dietas hiperproteicas se justifica por las altas exigencias productivas, pero a su vez, genera residuos por el metabolismo de la úrea, alterando el $\mathrm{pH}$ uterino y la fertilidad (Butler, 2000). Además, se producen desequilibrios endocrinos relacionados con la IGF-1 asociada a la fertilidad (Daftary y Gore, 2005; López-Luzardo, 2009). Si estos efectos negativos ocurriesen durante la gestación, Matsuo, Morikawa, Hashimoto y Baratz (1986) mencionaron se producirían alteraciones en la organogénesis.

Frente a lo mencionado, este proyecto buscó evaluar el efecto de tres niveles dietarios de proteína (12 g/100 g PC, 17g/100g PC y $22 \mathrm{~g} / 100 \mathrm{~g}$ PC) sobre el número de crías nacidas, el peso de la camada al nacimiento y al destete, y la ganancia de peso total de la camada.

\section{MATERIAL Y MÉTODOS}

El proyecto se realizó en el Centro Experimental de la Facultad de Medicina Veterinaria y Zootecnia de la Universidad Peruana Cayetano Heredia en Lurín, Lima. La temperatura promedio fue de $22^{\circ} \mathrm{C}$ de día / $16^{\circ} \mathrm{C}$ por las noches y humedad relativa de $76 \%$.

Los animales usados fueron cuyes domésticos (Cavia porcellus) de raza Andina, debido a sus características reproductivas (Araníbar y Echevarría, 2014; Vigil, 1971). El $n$ final fue de 36 cuyes hembra, sin partos previos ( $>2$ meses; >542gramos) y 6 cuyes machos de fertilidad comprobada ( $>4$ meses; $>850$ gramos). Se tuvo la aprobación del Comité Institucional del Ética para el uso de animales de la Universidad Peruana Cayetano de Heredia (UPCH) bajo la constancia 048-05-17.

\section{Diseño Experimental}

Adaptación: Los cuyes fueron alojados durante dos semanas en pozas de cemento $(1.2 \times 0.8 \times 1 \mathrm{~m})$ para aclimatarse a la localidad, alimentados con ración comercial y forraje fresco de maíz (Zea mays) cortado a los 45 días.

Grupos y tratamientos experimentales: Se usaron jaulas individuales $(0.6 \times 0.3 \times 0.25 \mathrm{~m})$ para poder alojarlos y dividirlos aleatoriamente en tres grupos experimentales (G1, G2 y G3), conformados por 12 hembras por grupo. El G1 recibió la dieta hipoproteica (12g/100g); el G2, la dieta normoproteica (17 g/100g); y el G3, la hiperproteica (22 g/100g)

Alimentación y formulación: La alimentación se basó en forraje fresco de maíz y las dietas experimentales, cuya formulación se llevó a cabo en el Programa de Proyección e Investigación Social en Alimentos de la Universidad Nacional Agraria de La Molina (UNALMN). Para los valores de energía digestible (ED) se consideraron valores isocalóricos de $2.8 \mathrm{kcal} / \mathrm{kg}$ para cada dieta, además de niveles de Lisina $(1,2 \mathrm{~g} / 100 \mathrm{~g})$ y Metionina $(0,3 \mathrm{~g} / 100 \mathrm{~g})$ para cada dieta (Torres, 2007). La formulación de las dietas experimentales se detalla en la tabla 1 y la composición del forraje en la tabla 2.

\section{Fases experimentales}

Cada grupo experimental recibió la dieta experimental compuesta por 50 gr de la ración correspondiente, 200 gr de forraje fresco (Zea mays) y agua ad libitum. Se consideró la inclusión del forraje por el aporte de fibra y vitamina $\mathrm{C}$.

La distribución de la alimentación se realizó de acuerdo a Terry et al., (2005) y se detalla a continuación:

- Pre-empadre: Fase constituida por 16 días, debido a la duración fisiológica de un ciclo estral completo, donde se proporcionó la dieta experimental correspondiente a cada grupo, incluyendo el forraje y agua ad libitum.

- Empadre: Fase constituida por 32 días, en donde cada grupo recibió la dieta experimental durante un periodo diurno, comprendido desde las $8 \mathrm{hrs}$ hasta las 14 horas. Posteriormente, entre las 14 horas y 8 horas de la mañana del día siguiente, las hembras fueron colocadas en la jaula del macho, con una 
Tabla 1. Análisis comparativo de los ingredientes y valores proximales de las dietas hipoproteica $(12 \mathrm{~g} / 100 \mathrm{~g})$, normoproteica $(17 \mathrm{~g} / 100 \mathrm{~g})$ e hiperproteica $(22 \mathrm{~g} / 100 \mathrm{~g})$

\begin{tabular}{cccc}
\hline & & Dietas \\
MATERIA PRIMA & Hipoproteica & Normoproteica & Hiperproteica \\
\cline { 2 - 4 } & \multicolumn{3}{c}{ PARTICIPACIÓN* (\%) } \\
\hline Afrecho de trigo & 75,51 & 65 & 57,84 \\
Maíz grano & 20,39 & 16,5 & 10,19 \\
Carbonato de calcio & 2,44 & 3,01 & 3,43 \\
Torta de soya 47\% pc & - & 14,35 & 26,54 \\
Hcl-lisina 78\% & 0,89 & 0,45 & - \\
Sal industrial & 0,5 & 0,5 & 0,5 \\
Premezcla vit-min & 0,1 & 0,1 & 0,1 \\
D-1-metionina 98\% & 0,14 & 0,07 & - \\
Harina de pescado 69\% pc & - & - & 1,03 \\
Harina de alfalfa & - & - & 0,34 \\
COMPOSICIÓN NUTRICIONAL (g/100g) & & $17 \mathrm{~g}$ & $22 \mathrm{~g}$ \\
Proteína cruda C) & $12 \mathrm{~g}$ & $7,30 \mathrm{~g}$ & $7 \mathrm{~g}$ \\
Fibra cruda (Fc) & $7,9 \mathrm{~g}$ & $1,20 \mathrm{~g}$ & $1,50 \mathrm{~g}$ \\
Calcio (Ca) & $1 \mathrm{~g}$ & $0,70 \mathrm{~g}$ & $0,80 \mathrm{~g}$ \\
Fósforo total (Pt) & $0,80 \mathrm{~g}$ & $1,20 \mathrm{~g}$ & $1,20 \mathrm{~g}$ \\
Lisina (Lys) & $1 . \mathrm{g}$ & $0,30 \mathrm{~g}$ & $0,30 \mathrm{~g}$ \\
Metionina (Met) & $0,30 \mathrm{~g}$ & $2800 \mathrm{kcal} / \mathrm{kg}$ & \\
Energía digestible (Ed) & & & \\
\hline
\end{tabular}

* Ingredientes mostrados de acuerdo a su participación real (gramos) en la pre-mezcla.

Tabla 2. Composición nutricional forraje fresco.

\begin{tabular}{ccccc}
\hline Presentación & Materia seca & Proteina cruda & Fibra & Ceniza \\
\hline Hojas (45 días) & 24,1 & 2,1 & 6,2 & 1,6 \\
\hline
\end{tabular}

Tabla 3. Resumen de las fases experimentales

\begin{tabular}{ccccc}
\hline Características & Pre empadre & Empadre & Gestación & Lactación \\
\hline Duración de fase (días) & 16 & 32 & Hasta el parto & 14 \\
Disponibilidad de dieta & $24 \mathrm{~h}$ & $8-14 \mathrm{~h}$ & $24 \mathrm{~h}$ & $24 \mathrm{~h}$ \\
Dieta Experimental & $50 \mathrm{~g}$ & $50 \mathrm{~g}$ & $50 \mathrm{~g}$ & $50 \mathrm{~g}$ \\
Consumo Dieta Experimental (\%) & $100 \%$ & $100 \%$ & $100 \%$ & $100 \%$ \\
Suministro de Chala & & & $24 \mathrm{~h}$ & $24 \mathrm{~h}$ \\
(Zea mays) & $24 \mathrm{~h}$ & $14-8 \mathrm{~h}$ & & Ad libitum \\
Agua & Ad libitum & $14-8 \mathrm{~h}$ & Ad libitum
\end{tabular}


Tabla 4. Resultados comparativos de los promedios estadísticos obtenidos para TC, PCN, PCD y GPT utilizando las dietas hipoproteicas, normoproteica e hiperproteica.

\begin{tabular}{|c|c|c|c|c|c|c|c|c|c|}
\hline \multirow{2}{*}{$\begin{array}{c}\text { Dieta } \\
\text { Experimental }\end{array}$} & \multicolumn{3}{|c|}{ Tamaño de camada } & \multicolumn{2}{|c|}{$\begin{array}{l}\text { Peso camada al } \\
\text { nacimiento (g) }\end{array}$} & \multicolumn{2}{|c|}{$\begin{array}{c}\text { Peso camada al } \\
\text { destete (g) }\end{array}$} & \multicolumn{2}{|c|}{$\begin{array}{c}\text { Ganancia peso } \\
\text { total (g/día) }\end{array}$} \\
\hline & $\mathbf{n}$ & $\ddot{\mathbf{X}}$ & Mediana & $\mathbf{n}$ & $\ddot{\mathbf{X}} \pm \mathbf{D S}$ & $\mathbf{n}$ & $\ddot{\mathbf{X}} \pm \mathbf{D S}$ & $\mathbf{n}$ & $\ddot{\mathbf{X}} \pm \mathrm{DS}$ \\
\hline Hipoproteica & 10 & $2^{\mathrm{a}}$ & 2 & 9 & $254,2 \pm 95,9^{\mathrm{a}}$ & 9 & $425,3 \pm 127,5^{\mathrm{a}}$ & 9 & $6,5 \pm 1,6^{\mathrm{a}}$ \\
\hline Normoproteica & 10 & $2,2^{\mathrm{a}}$ & 2 & 8 & $311,7 \pm 87,1^{\mathrm{a}}$ & 8 & $587,2 \pm 83,2^{\mathrm{b}}$ & 8 & $9,8 \pm 0,3^{\mathrm{b}}$ \\
\hline Hiperproteica & 11 & $1,9^{\mathrm{a}}$ & 2 & 9 & $230,9 \pm 74,9^{a}$ & 9 & $401,6 \pm 145,0^{\mathrm{a}}$ & 9 & $6,4 \pm 1.0^{\mathrm{a}}$ \\
\hline
\end{tabular}

a,b Letras diferentes en la misma columna, indican que hay diferencia significativa $(\mathrm{p}<0.05)$

* DS: Desviación Estándar

densidad de 1: 6, (Espinoza y Dorregaray, 1988; Solórzano y Sarria, 2014), en este periodo solo recibían forraje y agua. Luego, eran devueltas a sus jaulas individuales para consumir nuevamente la dieta experimental.

- Gestación: Acabado el empadre, las hembras fueron separadas en sus jaulas individuales y se les proporcionó la dieta experimental, forraje y agua ad libitum. Se esperó hasta el final de la gestación.

- Lactación: Se colocó a las madres de manera individual hasta el destete (14 días). Durante este periodo se proporcionó la dieta experimental, forraje y agua.

El resumen de las fases experimentales se presenta en la tabla 3.

\section{Variables evaluadas}

Las variables evaluadas en el estudio fueron las siguientes:

- Tamaño Camada (TC): Conteo de las crías nacidas por hembra.

- Peso camada al nacimiento (PCN): Peso total de los gazapos nacidos por hembra.

- Peso camada al destete (PCD): Peso de la camada a la segunda semana (14 días) de nacidos.

- Ganancia peso total (GPT): Obtenido por la diferencia de peso entre el PCD y el PCN, posteriormente este valor obtenido se dividió entre el número de crías nacidas por madre y finalmente dividido entre los 14 días.

Las variables cuantitativas discretas fueron descritas mediante la media aritmética y mediana; mientras que las variables cuantitativas continuas a través de media aritmética y desviación estándar. Las diferencias entre las medias de cada variable fueron evaluadas mediante Análisis de Varianza de 1 vía. Para la comparación múltiple de medias, se utilizó el test de Bonferroni. El análisis de datos se realizó utilizando el Software Stata versión 13,0 y se consideró un valor de 0,05 de significancia.

\section{RESULTADOS}

Durante las diferentes fases experimentales murieron dos hembras del grupo de dieta hipoprotieca, dos de la dieta hiperproteica y una de la dieta normoproteica. Por lo tanto, para el número de crías nacidas, se trabajó con 10 hembras para la dieta hipoproteica e hiperproteica, mientras que con 11 animales para la dieta normoproteica. El tamaño de camada y el peso de camada al nacimiento no presentaron diferencia estadística significativa $(p<0,05)$ por efecto del tipo de dieta. Por otro lado En el peso de camada al destete (14 días), la dieta normoproteica $17 \mathrm{~g} / 100 \mathrm{~g}$ tuvo mejores resultados y consecuentemente, gazapos con mejores ganancias de peso totales $(\mathrm{p}<0,05)$ (tabla 3 y tabla 4$)$.

\section{DISCUSIÓN}

Estudios realizados por el Instituto Nacional de Innovación Agraria [INIA] (2005) demuestran que los cuyes raza Andina, presentan un promedio de 2,9 crías al primer parto, el cual es mayor a los promedios obtenidos en este trabajo (hipoproteica: 2.0, normoproteica: 2,18 e hiperproteica: 1,91). Esta diferencia podría atribuirse al número de animales por grupo experimental y también a factores externos como el estrés propio del manejo durante las diferentes fases, lo que podría haber interrumpido la fase de implantación que ocurre de 5 a 7 días post-cópula (Blandau, 1949).

No existe diferencia significativa $(p<0,05)$ entre los pesos de camada al nacimiento por efecto de 
los diferentes niveles de proteína. La malnutrición durante la gestación y lactación, provoca que los fetos presenten mecanismos compensatorios para protección de órganos vitales, reduciendo su metabolismo y aprovechando mejor los nutrientes disponibles; sin embargo, esto sí podría causar problemas endocrinos en la progenie (Moura et al., 2008; Zambrano et al., 2005). La disponibilidad de forraje fresco y tener reducida actividad física podría haber disminuido el gasto calórico de las cuyes compensando la poca ingesta proteica (Fidalgo et al., 2012).

Los pesos al nacimiento fueron mayores a lo reportado por el INIA (2005) (115 g). Estas diferencias fueron demostradas previamente por Ordoñez (1997), quienes encontraron que a mayor número de crías nacidas, menor era el peso individual de los gazapos y mayor el porcentaje de mortalidad durante la lactancia. Consecuentemente, en este experimento se obtuvo un menor número de camada que el usual para la raza Andina, por ende se logró mayores pesos al nacimiento.

Los resultados demostraron que las madres alimentadas con dietas normoproteicas tuvieron gazapos con mejor pesos total al destete en comparación con las otras dietas ofrecidas. Esto refuerza la idea que explotaciones cuyas dietas tengan un desbalance en las fuentes proteicas, tendrán animales de menor peso final y por lo tanto, carcasas de menor peso.

El porcentaje proteico dietario influye sobre el mantenimiento de las funciones fisiológicas de la madre y los fetos. Sin embargo, durante la gestación los fetos podrían modular la organogénesis para compensar cuadros metabólicos como la desnutrición o el exceso de desechos nitrogenados, resultado de dietas hipo e hiperproteicas, respectivamente (Saravia et al., 1983; Matsuo et al., 1986). A pesar de esto, las modulaciones fetales podrían causar un efecto retardado en el descenso testicular, la pubertad y aumentar el ratio folículos vesiculares: folículos de Graaf (Leonhardt et al., 2003).

Asimismo, las dietas hipoproteicas, estarían produciendo un cuadro de subnutrición porque el porcentaje proteico necesario durante la gestación y lactación es de mínimo $18 \mathrm{~g} / 100 \mathrm{~g}$. Se conoce que la restricción proteica durante la gestación genera una priorización de los nutrientes maternos para mantener las funciones y órganos vitales. Sin embargo, a la vez produce efectos deletéreos sobre la progenie, principalmente retrasa el desarrollo gonadal, producción de hormonas y disminuye de los niveles de leptina, retrasando así la pubertad en animales jóvenes debido una baja producción de gonadotropinas (Clarke y Henry, 1999; Da Silva, Brasil, Sampaio y da Fonte, 2008; Zambrano et al., 2005).

En el otro escenario planteado, las madres alimentadas con altos valores proteicos también presentaron gazapos con pesos inferiores al destete, coincidiendo con lo encontrado por Ordoñez (1997), quien reporta mejores pesos y ganancias diarias usando dietas normo proteicas $(17 \mathrm{~g} / 100 \mathrm{~g})$ en comparación con hiperproteicas $(21 \mathrm{~g} / 100 \mathrm{~g})$.

La mejor ganancia de peso total fue utilizando la dieta normoproteica, indicando que los excesos proteicos en las dietas no siempre generan un efecto positivo sobre las camadas. Incluso no se encontraron diferencias significativas $(\mathrm{p}<0.05)$ en las ganancias de peso entre las dietas hipo e hiperproteicas. Por lo tanto, esto podría sugerir a los pequeños productores la idea de que los niveles proteicos idóneos (17-18g/100g) podrían contribuir a mejorar los índices productivos.

\section{Correspondencia:}

\section{Carlos Paco Nagaki}

Correo electrónico: carlospaconagaki@gmail.com

\section{REFERENCIAS BIBLIOGRAFICAS}

1. Araníbar, E., \& Echevarría C., L. (2014). Número de ovulaciones por ciclo estrual en cuyes (Cavia porcellus) Andina y Perú. Revista De Investigaciones Veterinarias del Perú, 25(1), 29-36.

2. Blandau, R. J. (1949), Observations on implantation of the guinea pig ovum. Anat. Rec, 103, 19-47. doi:10.1002/ar.1091030103

3. Butler, W.R. (2000). Nutritional interactions with reproductive performance in dairy cattle. Anim Reprod Sci, 60-61,449-457.

4. Camolezi, A.L. (2011). Desenvolvimento sexual da prole de ratas submetidas à restrição proteica em diferentes periodos da prenhez e lactação. (Dissertação de mestrado. U n i v e r s i d a d e Estadual Paulista, São Paulo, Brasil).

5. Chauca, L. (1997). Crianza de Cuyes (Cavia porcellus). Roma: Organización de las Naciones Unidas para la Agricultura y la Alimentación.

6. Clarke, I.J. \& Henry, B.A. (1999). Leptin and reproduction. Rev Reprod, 4, 48-55.

7. Da Silva Faria, T., Brasil, F.B., Sampaio F.J.B. \& da Fonte Ramos C. (2008). Maternal malnutrition during lactation alters the folliculogenesis and gonadotropins 
and estrogen isoforms ovarian receptors in the offspring at puberty. J Endocrinol, 198, 625-634.

8. Daftary S. \& Gore C (2005). IGF-1 in the brain as a regulator of reproductive neuroendocrine function. Exp Biol Med (Maywood), 230, 292-306.

9. Espinoza, F. \& Dorregaray, H. (1994) Evaluación de parámetros productivos en cuyes de acuerdo a la densidad por jaula. En: Instituto Nacional de Investigación Agraria. Investigaciones en cuyes. Junín, Perú: Universidad Nacional del Centro.

10. Fidalgo, M., Falcão-Tebas F., Bento-Santos A., de Oliveira E., Nogueira-Neto J.F., de Moura E.G...

11. Leandro, C.G. (2012). Programmed changes in the adult rat offspring caused by maternal protein restriction during gestation and lactation are attenuated by maternal low physical training. Br J Nutr, 1,1-8.

12. Instituto Nacional de Innovación Agraria. (2005). Cuyes Raza Andina. Lima: Ministerio de Agricultura. Recuperado de: http://www.inia.gob.pe/wp-content/ uploads/investigacion/programa/sistProductivo/raza/ cuy/Cuy-raza-andina.pdf

13. Leonhardt, M., Lesage, J., Croix, D., DutriezCasteloot, I., Beauvillain, JC. \& Dupouy, J.P. (2003). Effects of perinatal maternal food restriction on pituitarygonadal axis and plasma leptin level in rat pup at birth and weaning and on timing of puberty. Biol Reprod, 68,390-400.

14. López-Luzardo, M. (2009). Las dietas hiperproteicas y sus consecuencias metabólicas. Anales Venezolanos de Nutrición, 22(2), 95-104

15. Matsuo, M., Morikawa, Y., Hashimoto, Y. \& Baratz, R.S. (1986). Changes in blood urea nitrogen (BUN) concentration during pregnancy in the rat with or without obstructive uremia. Exp Pathol;30:203e8.

16. Moura, E.G., Santos, R.S., Lisboa, P.C., Alves, S.B., Bonomo, I.T., Fagundes, A.T... Passos, M.C. (2008).

17. Thyroid function and body weight programming by neonatal hyperthyroidism in rats - the role of leptin and deiodinase activities. Horm Metab Res, 40, 1-7.
18. Ordoñez, R. (1997). Efecto de dos niveles de proteína y fibra cruda en el alimento de cuyes (Cavia porcellus) en lactación y crecimiento. (Tesis de grado Ingeniero Zootecnista. Universidad Agraria de la Molina, Lima, Perú).

19. Saravia, D.J., Muscari, G.J. \& Chauca, F.L. (1983). Flushing en cuyes hembras en reproducción. VI Reunión científica anual de la Asociación Peruana de Producción Animal (APPA). Lambayeque, Perú.

20. Solórzano, J. \& Sarria, J. (2014). Crianza, producción y comercialización de cuyes. Lima, Perú: Colección Agro, Editorial Macro.

21. Terry, K.K., Chatman, L.A., Foley, G.L., Kadyszewski, E., Fleeman, T.L., Hurtt, M.E. \& Chapin, R.E. (2005). Effects of feed restriction on fertility in female rats. Birth Defects Res B Dev Reprod Toxicol, 74(5), 43141.

22. Torres, A. (2007). Evaluación de dos niveles de energía y proteína en el concentrado de crecimiento para cuyes machos. (Tesis de Ingeniero Zootecnista.

23. Universidad Nacional Agraria de la Molina, Lima, Perú).

24. Vigil, D.V. (1971). Caracterización del ciclo eustral en cobayos hembras vírgenes (Cavia porcellus). (Tesis de Ingeniero Zootecnista. Universidad Nacional Agraria de la Molina, Lima, Perú).

25. Wheat, J.D., Spies, H.G., Tran C.T. \& Kock, B.A. (1962). Effects of two protein levels on growth rate and feed efficiency of guinea pigs from different inbred lines. J Anim Sci, 22, 670-673.

26. Zaldívar, A.M. (1986). Estudio de la edad de empadre de cuyes hembras (Cavia porcellus) y su efecto sobre el tamaño y peso de camada. (Tesis de Ingeniero Zootecnista. Universidad Nacional Agraria de la Molina, Lima, Perú).

27. Zambrano, E., Rodriguez-Gonzalez, GL., Guzman, C., García-Becerra, R., Boeck, L., Diaz, L... Nathanielsz, P.W. (2005). A maternal low protein diet during pregnancy and lactation in the rat impairs male reproductive development. J Physiol, 563,275-284. 